Microbiol Rev. 2013;26:657-702. 1 http://dx.doi.org/10.1128/ CMR.00032-13

8. Karasartova D, Gureser AS, Gokce T, Celebi B, Yapar D, Keskin A, et al. Bacterial and protozoal pathogens found in ticks collected from humans in Corum province of Turkey. PLoS Negl Trop Dis. 2018;12:e006395. http://dx.doi.org/10.1371/journal.pntd.0006395

9. Tosoni A, Mirijello A, Ciervo A, Mancini F, Rezza G, Damiano F, et al.; Internal Medicine Sepsis Study Group. Human Rickettsia aeschlimannii infection: first case with acute hepatitis and review of the literature. Eur Rev Med Pharmacol Sci. 2016;20:2630-3.

10. Boldbaatar B, Jiang RR, von Fricken ME, Lkhagvatseren S, Nymadawa P, Baigalmaa B, et al. Distribution and molecular characteristics of rickettsiae found in ticks across Central Mongolia. Parasit Vectors. 2017;10:61. http://dx.doi.org/10.1186/ s13071-017-1981-3

Address for correspondence: Norio Ohashi, University of Shizuoka, Laboratory of Microbiology, Department of Food Science and Biotechnology, School of Food and Nutritional Sciences, Graduate School of Integrated Pharmaceutical and Nutritional Sciences, 52-1 Yada, Suruga-ku, Shizuoka 422-8526, Japan; email: ohashi@u-shizuoka-ken.ac.jp

\title{
Japanese Spotted Fever in Eastern China, 2013
}

\section{Jiabin Li, ${ }^{1}$ Wen Hu, ${ }^{1}$ Ting Wu, Hong-Bin Li, Wanfu Hu, Yong Sun, Zhen Chen, Yonglin Shi, Jia Zong, Adams Latif, Linding Wang, Li Yu, Xue-Jie Yu, Bo-Yu Liu, Yan Liu}

\begin{abstract}
Author affiliations: The First Affiliated Hospital of Anhui Medical University, Hefei, China (J. Li, T. Wu, H.-B. Li); The First Affiliated Hospital of the University of Science and Technology of China, Hefei (Wen Hu); Anhui Center for Disease Control and Prevention, Hefei (Wanfu Hu, Y. Sun, Y. Shi); Anhui Medical University, Hefei (Z. Chen, J. Zong, A. Latif, L. Wang, L. Yu, B.-Y. Liu, Y. Liu); Wuhan University School of Health Sciences, Wuhan, China (X.-J. Yu)
\end{abstract}

DOI: https://doi.org/10.3201/eid2411.170264

We isolated Rickettsia japonica from a febrile patient in Lu'an City, China, in 2013. Subsequently, we found an $R$. japonica seroprevalence of $54.8 \%(494 / 902)$ in the rural population of Anhui Province and an R. japonica prevalence in Haemaphysalis longicornis ticks of $0.5 \%$ (5/935). $R$. japonica and its tick vector exist in China.

${ }^{1}$ These authors contributed equally to this article.
Q potted fever group rickettsiae are tickborne, obliga$\checkmark$ tory intracellular, gram-negative bacteria with a worldwide distribution. However, the distribution of each species of spotted fever group rickettsiae is limited to geographic areas by their specific tick vectors. Japanese spotted fever is a severe rickettsiosis caused by Rickettsia japonica bacterium $(1,2)$, which has been present in Japan since 1984 and isolated from patients in other countries of Asia (e.g., South Korea, the Philippines, and Thailand) over the past decade $(3,4)$. In this study, we present information on an $R$. japonica isolate acquired from a febrile patient and $R$. japonica seroprevalence in Anhui Province in eastern China.

On August 7, 2013, a 61-year-old man from Shucheng County, Lu'an City, China, in the Dabie Mountain area of Anhui Province (online Technical Appendix Figure 1, https:// wwwnc.cdc.gov/EID/article/24/11/17-0264-Techapp1. pdf) with fever and headache for 1 week was admitted into Shucheng County People's Hospital. The patient reported several tick bites 10 days before the onset of his illness. At admission, the patient was conscious and had fever $\left(39.0^{\circ} \mathrm{C}\right)$; he did not have jaundice, and no bleeding was found on his skin or mucosal membranes. A papular rash with papules $0.1-0.5 \mathrm{~cm}$ in diameter was noted all over his body (online Technical Appendix Figure 2). Blood cell counts showed the patient had leukocytosis $\left(10.34 \times 10^{9}\right.$ cells/L), increased neutrophils $(87.5 \%)$, and a platelet count within reference range $\left(130 \times 10^{9} / \mathrm{L}\right)$. Blood chemistry testing revealed a urea nitrogen concentration of $9.12 \mathrm{mmol} / \mathrm{L}$ (reference range 2.9-8.2 $\mathrm{mmol} / \mathrm{L})$, creatinine of $0.758 \mathrm{mg} / \mathrm{dL}(67 \mu \mathrm{mol} / \mathrm{L}$, reference range $53-106 \mu \mathrm{mol} / \mathrm{L}$ ), C-reactive protein of $77.5 \mathrm{nmol} / \mathrm{L}$ (reference range $0.76-28.5 \mathrm{nmol} / \mathrm{L}$ ), and an erythrocyte sedimentation rate of $22 \mathrm{~mm} / \mathrm{h}$ (reference range $0-20 \mathrm{~mm} / \mathrm{h}$ ). A urine test showed a procalcitonin concentration of $0.806 \mathrm{ng} /$ $\mathrm{mL}$ (reference range $\leq 0.15 \mathrm{ng} / \mathrm{mL}$ ) and an interleukin 6 concentration of $52 \mathrm{pg} / \mathrm{mL}$ (reference range $\leq 1.8 \mathrm{pg} / \mathrm{mL}$ ). The patient had rough lung breath sounds, and computed tomography showed inflammatory infiltrates in the middle right lung and lower left lung lobe, bullae on the upper left lung lobe, and emphysematous changes. The patient was suspected to have a rickettsial infection and was given minocycline and meropenem on the day of his admission. Two days later, on August 9,2013 , the patient's fever subsided $\left(36.2^{\circ} \mathrm{C}\right)$, and he was discharged.

A blood sample taken from the patient 1 day after admission was inoculated onto THP-1 and Vero E6 cells; after 10 days, cytopathic effect was visible by light microscopy with only the THP-1 cells. Diff-Quick (Thermo Fisher Scientific, Kalamazoo, MI, USA)-stained smears of THP-1 cells showed Rickettsia-like bacilli in the cytoplasm. Electron microscopy showed the bacilli localized to the cytoplasm and nucleus and had the typical ultrastructure of Rickettsia bacteria. This species was highly pleomorphic 
but mainly had dimensions $0.2 \mu \mathrm{m} \times 0.5-1 \mu \mathrm{m}$ (online Technical Appendix Figure 3).

We amplified and sequenced the $17-\mathrm{kDa}$ protein gene, 16S rRNA gene, ompA, ompB, and gene $\mathrm{D}$ of $R$. $j a$ ponica (GenBank accession nos. KY364904, KY484160, KY484162, KY484163, and KY488633; online Technical Appendix Table). These gene sequences were $99.8 \%-100 \%$ homologous with the corresponding gene of an $R$. japonica isolate (GenBank accession no. AP017602.1).

Hard-body tick species Haemaphysalis longicornis, H. flava, and Dermacentor taiwanensis $(5,6)$ have been reported as $R$. japonica transmission vectors. We acquired questing $H$. longicornis ticks in Shandong Province, China, in 2013 and found them positive for the R. japonica $17-\mathrm{kDa}$ protein and 16S rRNA genes by PCR (online Technical Appendix). The percentage of $H$. longicornis ticks infected with $R$. japonica rickettsia in Shandong Province was $0.5 \%$ (5/975). The H. longicornis tick, which is prevalent in East China and feeds on domestic animals and small mammals, might be a major vector of $R$. japonica rickettsia in China
$(7,8)$. Phylogenic analysis of the 16S rRNA (Figure, panel $\mathrm{A}$ ) and $17-\mathrm{kDa}$ protein (Figure, panel B) genes indicated that the rickettsial isolates from the patient and $H$. longicornis tick were identical to $R$. japonica isolates and in the same clade with $R$. heilongjiangensis.

Examination by indirect immunofluorescence assay showed that the patient's acute (1:80 dilution) and convalescent (1:1,280 dilution) serum samples reacted to isolated antigen of $R$. japonica bacterium. During 2013, we collected serum samples from 902 healthy persons living in rural areas of Anhui Province (online Technical Appendix Figure 1 ) and tested them with the same assay. We found $54.8 \%$ (494/902) of serum samples positive for $R$. japonica-specific antibodies.

In summary, we detected $R$. japonica bacteria in a patient and an $H$. longicornis tick and demonstrated high $R$. japonica seroprevalence among the rural population of Anhui Province. In agreement with $\mathrm{Lu}$ et al.'s work in 2015 (9), our findings suggest that $R$. japonica might be more prevalent in China than previously thought. Physicians in

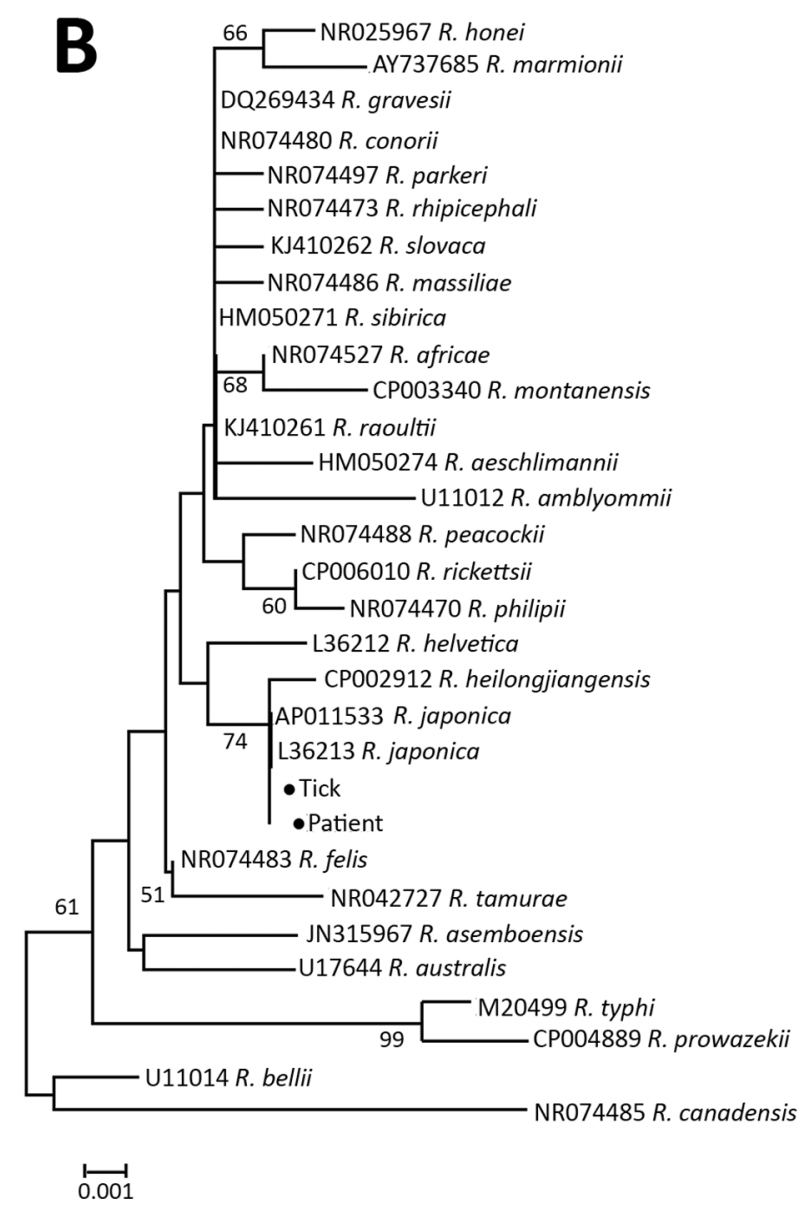

Figure. Phylogenetic analysis of Rickettsia isolate from patient with Japanese spotted fever in Anhui Province and isolate from Haemaphysalis longicornis tick in Shandong Province, China, 2013 (black dots), compared with reference isolates. Unrooted neighborjoining trees of $16 \mathrm{~S}$ rRNA gene (A) and 17-kDa protein gene (B) were constructed by using MEGA 5.2 (https://www.megasoftware.net/) and 1,000 bootstrap replications. Scale bars represent substitutions per nucleotide. 
China need to become aware of $R$. japonica disease presentation, so they can administer the appropriate treatment to patients with suspected $R$. japonica infections.

This study was supported by the National Natural Science Foundation of China (81571963); Science Foundation of Anhui Province of China (1608085MH213); Natural Science Foundation Key Project of Anhui Province Education Department (KJ2015A020, KJ2016A331); and Scientific Research of Anhui Medical University (XJ201314, XJ201430, XJ201503).

\begin{abstract}
About the Authors
Dr. Li is a research coordinator at The First Affiliated Hospital of Anhui Medical University, Hefei, China. His research interests are pathogenic mechanisms of tickborne infectious diseases, including severe fever with thrombocytopenia syndrome, human granulocytic anaplasmosis, and spotted fever group rickettsioses. Dr. Wen $\mathrm{Hu}$ is an electron microscope technician at The First Affiliated Hospital of the University of Science and Technology of China, Hefei, China. His research interest is pathogen structure.
\end{abstract}

\section{References}

1. Uchida T, Yu XJ, Uchiyama T, Walker DH. Identification of a unique spotted fever group rickettsia from humans in Japan. J Infect Dis. 1989;159:1122-6. http://dx.doi.org/10.1093/ infdis/159.6.1122

2. Mahara F. Japanese spotted fever: report of 31 cases and review of the literature. Emerg Infect Dis. 1997;3:105-11. http://dx.doi.org/10.3201/eid0302.970203

3. Chung MH, Lee SH, Kim MJ, Lee JH, Kim ES, Lee JS, et al. Japanese spotted fever, South Korea. Emerg Infect Dis. 2006; 12:1122-4. http://dx.doi.org/10.3201/eid1207.051372

4. Gaywee J, Sunyakumthorn P, Rodkvamtook W, Ruang-areerate T, Mason CJ, Sirisopana N. Human infection with Rickettsia sp. related to R. japonica, Thailand. Emerg Infect Dis. 2007;13:657-9. http://dx.doi.org/10.3201/eid1304.060585

5. Uchida T, Yan Y, Kitaoka S. Detection of Rickettsia japonica in Haemaphysalis longicornis ticks by restriction fragment length polymorphism of PCR product. J Clin Microbiol. 1995;33:824-8.

6. Ishikura M, Fujita H, Ando S, Matsuura K, Watanabe M. Phylogenetic analysis of spotted fever group rickettsiae isolated from ticks in Japan. Microbiol Immunol. 2002;46:241-7. http://dx.doi.org/10.1111/j.1348-0421.2002.tb02692.x

7. Luo LM, Zhao L, Wen HL, Zhang ZT, Liu JW, Fang LZ, et al. Haemaphysalis longicornis ticks as reservoir and vector of severe fever with thrombocytopenia syndrome virus in China. Emerg Infect Dis. 2015;21:1770-6. http://dx.doi.org/10.3201/ eid2110.150126

8. Tenquist J, Charleston W. A revision of the annotated checklist of ectoparasites of terrestrial mammals in New Zealand. J R Soc N Z. 2001;31:481-542. http://dx.doi.org/10.1080/03014223.2001.9517666

9. Lu Q, Yu J, Yu L, Zhang Y, Chen Y, Lin M, et al. Rickettsia japonica infections in humans, Zhejiang Province, China, 2015. Emerg Infect Dis. 2018 Nov [date cited]. https://doi.org/10.3201/ eid2411.170044

Address for correspondence: Yan Liu, Anhui Medical University, School of Basic Medical Sciences, Anhui, 230032, China; email: yliu16888@163.com; Bo-Yu Liu; email: centian2004@163.com

\section{Burkholderia lata Infections from Intrinsically Contaminated Chlorhexidine Mouthwash, Australia, 2016}

\author{
Lex E.X. Leong, Diana Lagana, Glen P. Carter, \\ Qinning Wang, Kija Smith, Tim P. Stinear, \\ David Shaw, Vitali Sintchenko, \\ Steven L. Wesselingh, Ivan Bastian, \\ Geraint B. Rogers
}

Author affiliations: South Australian Health and Medical Research Institute, Adelaide, South Australia, Australia (L.E.X. Leong, S.L. Wesselingh, G.B. Rogers); Flinders University, Bedford Park, South Australia, Australia (L.E.X. Leong, G.B. Rogers); Royal Adelaide Hospital, Adelaide (D. Lagana, D. Shaw); University of Melbourne, Melbourne, Victoria, Australia (G.P. Carter, T.P. Stinear); The University of Sydney, Westmead, New South Wales, Australia (Q. Wang, V. Sintchenko); SA Pathology, Adelaide (K. Smith, I. Bastian)

DOI: https://doi.org/10.3201/eid2411.171929

Burkholderia lata was isolated from 8 intensive care patients at 2 tertiary hospitals in Australia. Whole-genome sequencing demonstrated that clinical and environmental isolates originated from a batch of contaminated commercial chlorhexidine mouthwash. Genomic analysis identified efflux pump-encoding genes as potential facilitators of bacterial persistence within this biocide.

$B$ urkholderia contaminans and B. lata together form group $\mathrm{K}$ of the B. cepacia complex (Bcc). These predominantly environmental species are a major cause of pharmaceutical contamination and have been linked to multiple instances of associated opportunistic infection (1). Although both species are capable of causing serious infections in humans $(2,3)$, only $B$. contaminans has been associated with infection outbreaks $(3,4)$. We report a healthcareassociated $B$. lata infection outbreak occurring in intensive care units (ICUs) in 2 tertiary hospitals in Australia.

During May-June 2016, bacterial contamination of chlorhexidine mouthwash $(0.2 \% \mathrm{mg} / \mathrm{mL})$ was associated with an interjurisdictional outbreak in New South Wales and South Australia. Bcc isolates were obtained from blood and tracheal aspirates from 6 ICU patients in hospital A (South Australia) (sample information and isolation protocols detailed in the online Technical Appendix, https:// wwwnc.cdc.gov/EID/article/24/11/17-1929-Techapp1. pdf). An investigation by the hospital's infection and prevention control team noted discoloration of a commercial chlorhexidine mouthwash. Bcc isolates were cultured from 